\title{
CARACTERIZAÇÃO ESPECTRAL DE ESTÁGIOS SUCESSIONAIS DE VEGETAÇÃO SECUNDÁRIA ARBÓREA EM ALTAMIRA (PA), ATRAVÉS DE DADOS ORBITAIS ${ }^{1}$
}

\author{
Flávio Jorge Ponzoni², Ana Carolina Pinto Rezende ${ }^{2}$
}

\begin{abstract}
RESUMO - Imagens TM/Landsat 5 (bandas TM1-TM5 e TM7) de Altamira, PA, de 07.02.1991 foram transformadas em reflectância de superfície usando o modelo 6S de correção atmosférica com o objetivo de caracterizar espectralmente formações florestais secundárias. Dados biofísicos (DAP, G e H) foram coletados de 16 parcelas em campo, os quais foram utilizados no cálculo da área basal total (GT) e da rugosidade do dossel (Rug) e no estabelecimento de correlações entre os valores de reflectância de superfície dessas mesmas parcelas. Os coeficientes de correlação mais elevados foram verificados nas relações com a banda TM7. Regressões lineares foram estabelecidas tendo como variável dependente os parâmetros biofísicos e como variável independente, os valores de reflectância de superfície da banda TM7, a partir das quais foram elaborados mapas temáticos representativos da distribuição espacial dos parâmetros biofísicos. A reflectância de superfície não foi sensível a valores pequenos de dados biofísicos. Apesar disso, a metodologia aqui empregada mostrou-se eficaz para estimativas remotas de dados dendrométricos.
\end{abstract}

Palavras-chave: Caracterização espectral, estágios sucessionais, radiometria da vegetação.

\section{SPECTRAL CHARACTERIZATION OF SUCCESSIONAL STAGES OF VEGETATION IN ALTAMIRA (PA), BRAZIL USING ORBITAL RADIOMETRIC DATA}

\begin{abstract}
Landsat 5 TM images (TM1 to TM5 and TM7 spectral bands) from Altamira PA (Brazil) for 02.07.1991, were transformed into surface reflectance images by the 65 atmospheric correction code in order to characterize spectrally secondary succession stages of vegetation. Biophysical data $(A D B H, A B A, T B A$, THS and THSTD) collected in the field from 16 sampled vegetation plots were utilized in a correlation study between them and the surface reflectance values extracted from the orbital images. The highest correlation coefficients were found for the TM7 spectral band. Linear regression models were determined for each biophysical parameter considered and thematic images representing the spatial distribution of these parameters were made. The spectral data were not sensitive for detecting lower biophysical parameter values. Nevertheless, the results show that there was a good match between that spatial distribution and the study area landscape, indicating that these thematic images could be utilized in preliminary evaluations of the vegetation's biophysical characteristics.
\end{abstract}

Key Words: Spectral characterization, successional stages, vegetation radiometry.

\section{INTRODUÇÃO}

Estudos de desflorestamentos em ambiente tropical, particularmente na Amazônia brasileira, têm se concentrado na determinação de taxas desse uso humano da paisagem ao longo do tempo (MORAN et al., 2000).
Alves et al. (1999) comentaram que, como conseqüência do crescente interesse na determinação dessas taxas, tem crescido a atenção às caracterizações do uso da terra e da cobertura vegetal, nas quais o conhecimento da dinâmica sucessional da vegetação é fundamental. Adicionalmente, a vegetação secundária tem sido

\footnotetext{
${ }^{1}$ Recebido para publicação em 21.2.2003 e aceito para publicação em 10.8.2004.

${ }^{2}$ Instituto Nacional de Pesquisas Espaciais - Divisão de Sensoriamento Remoto. E.mail: <flavio@ ltid.inpe.br
} 
considerada elemento fundamental em estudos de seqüestro de carbono, sendo frequientemente necessária a estimativa da biomassa dessas formações vegetais (SOUSA e PONZONI, 1996).

As técnicas de sensoriamento remoto têm sido consideradas como alternativa na quantificação de biomassa florestal, uma vez que a radiação eletromagnética refletida por dosséis vegetais e registrada por sensores remotamente situados é fruto de um processo de interação que inclui a interferência de fatores espectrais dos elementos dessa vegetação (folhas, galhos, troncos etc.), geométricos de iluminação e de visada e de parâmetros biofísicos (distribuição espacial, densidade, biomassa etc.) da vegetação (PONZONI e DISPERATI, 1994). Vale salientar que o que é efetivamente registrado por esses sensores são valores de radiância que, como foi mencionado, são explicados pela comunhão de fatores de diferentes naturezas, sendo a biomassa florestal apenas um deles, ou seja, um único valor de radiância é explicado pela influência de inúmeros fatores, o que confere aos dados remotamente coletados um caráter ambíguo (GOEL, 1988; PONZONI, 2001). Apesar dessa limitação intrínseca à aplicação dos dados de sensoriamento remoto, as relações entre parâmetros biofísicos da vegetação e dados radiométricos orbitais podem, em alguns casos surpreender positivamente, dependendo da escala na qual os dados são coletados e correlacionados. É o caso dos resultados alcançados por Ponzoni et al. (1996), por ocasião do estabelecimento de correlações entre dados radiométricos coletados a bordo de um helicóptero e dados dendrométricos de plantios de Eucalyptus sp. colhidos em campo, com valores de coeficientes de correlação em torno de $80 \%$. Esses autores identificaram correlações consistentes também entre dados radiométricos extraídos de imagens orbitais do sensor Thematic Mapper (TM) do satélite Landsat 5, referentes aos mesmos plantios de Eucalyptus sp., e os dados dendrométicos mencionados.

Dentre as várias iniciativas de estudos no âmbito do Experimento de Grande Escala da Biosfera-Atmosfera da Amazônia (LBA), realizado a partir de acordos firmados entre instituições brasileiras e norte-americanas, destacam-se os estudos das formações florestais secundárias. No caso das aplicações das técnicas de sensoriamento remoto nessas pesquisas, estas não têm se restringido somente à sua identificação, incluindo também a quantificação de parâmetros biofísicos, como o indice de area foliar, rugosidade de dossel e biomassa.

O objetivo deste trabalho foi avaliar o potencial e as limitações do uso de dados radiométricos orbitais na caracterização espectral de vegetação secundária arbórea na região de Altamira, PA, a partir do estabelecimento de correlações entre parâmetros biofísicos coletados em campo e dados radiométricos extraídos de imagens orbitais TM/Landsat.

\section{MATERIAL E MÉTODOS}

\section{1. Área de estudo}

A área de estudo foi explorada previamente por Mausel et al. (1993) e situa-se no município de Altamira, PA, apresentando dimensões de aproximadamente 45 x 91 km e localizando-se ao longo da rodovia Transamazônica. As fitofisionomias dominantes incluem as florestas de terras firmes e as florestas com lianas. A precipitação anual é de aproximadamente $1.700 \mathrm{~mm}$, com quatro meses de período seco entre junho e setembro (MAUSEL et al., 1993). Na Figura 1, mostra-se a localização da área de estudo no contexto da região amazônica.

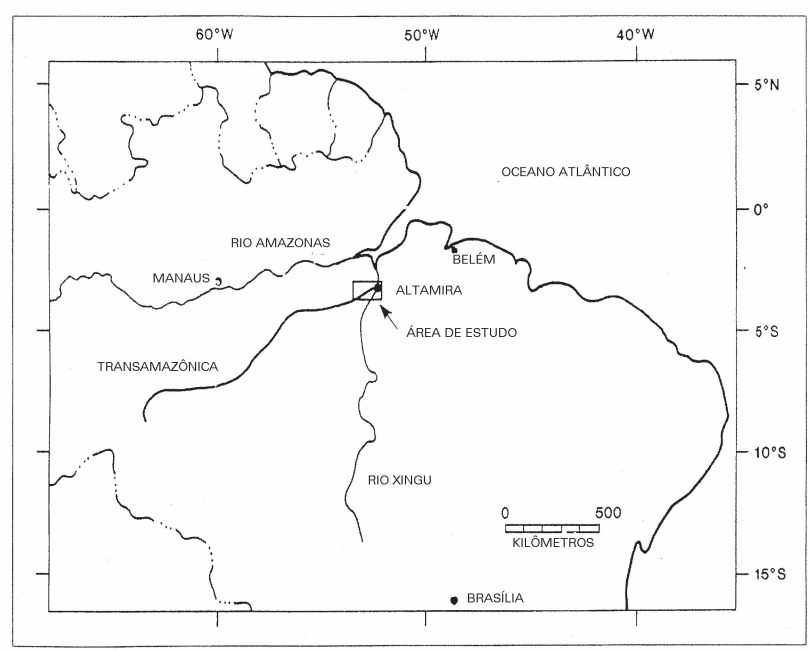

Fonte: (MAUSEL et al., 1993)

Figura 1 - Localização da área de estudo no contexto amazônico. Figure 1 - Study area location in an Amazonian context. 
A paisagem da área de estudo é caracterizada por uma rodovia principal (Transamazônica) e por outras secundárias perpendiculares à principal, estendendose a diferentes distâncias. Ao longo dessa rede de rodovias, pequenos agricultores têm plantado diferentes culturas agrícolas comerciais, sendo as pastagens o uso da terra dominante. Áreas abandonadas são também comuns, as quais são caracterizadas por vegetação em diferentes estágios sucessionais, os quais foram descritos por Mausel et al. (1993), considerando três níveis: estágio inicial (SS1), no qual ocorre vegetação graminóide dispersa entre vegetação de porte arbórea em crescimento; estágio intermediário (SS2), caracterizado pela presença de árvores entre 8 e 12 m de altura, com uma estratificação vertical relativamente evidente; e, finalmente, o estágio avançado (SS3), no qual o dossel apresenta uma estratificação vertical mais evidente do que em SS2 e com árvores normalmente excedendo $20 \mathrm{~m}$ de altura.

\subsection{Dados biofísicos}

Moran et al. (2000) descreveram a estratégia adotada na amostragem e coleta dos dados que foram conduzidas em trabalhos de campo em quatro diferentes áreas de estudo em 1993, incluindo Altamira, cujos dados foram aqui utilizados. Segundo procedimento descrito pelos autores, parcelas de $10 \times 15 \mathrm{~m}$ e subparcelas de $5 \times 2 \mathrm{~m}$ foram aleatoriamente distribuídas com o objetivo de extrair dados dendrométricos $(\mathrm{DAP}>=10 \mathrm{~cm})$, de arboretos (DAP entre $2-10 \mathrm{~cm}$ ), de mudas (DAP< $2 \mathrm{~cm}$ ) e de vegetação herbácea. Nas parcelas, todos os indivíduos arbóreos foram identificados floristicamente, sendo medidos o DAP, a altura comercial (HC) e a altura total (H). Nas subparcelas, todos os arboretos foram igualmente identificados floristicamente e tiveram DAP e $\mathrm{H}$ medidos, enquanto as mudas e vegetação herbácea foram também identificadas e contadas. Os dados das subparcelas não foram considerados neste trabalho.

A partir desses dados foram calculados a área basal individual (GI), através da aplicação da equação $\mathrm{GI}=\pi \mathrm{DAD}^{2} / 4$, para cada árvore em cada parcela. Esses valores individuais de GI foram, então, utilizados para calcular a área basal total (GT) por parcela, mediante a soma de todos os individuais valores de GI dentro de cada parcela; a área basal média $(\mathrm{G})$, através da relação entre GT e a área específica de cada parcela (as parcelas possuíam tamanhos diferentes).

Os valores de H e de DAP médio foram expressos pelas médias aritméticas dos valores individuais em cada parcela. O desvio-padrão dos valores de $\mathrm{H}$ em cada parcela foi utilizado para representar a rugosidade do dossel (Rug), que influencia a reflectância nas regiões espectrais do visível e do infravermelho (GOEL, 1988).

Foram considerados aqui os dados de 16 parcelas utilizadas por Mausel et al. (1993), sendo três delas caracterizadas pelo estágio SS1, oito pelo estágio SS2, três pelo estágio SS3, uma pela floresta primária de terra firma e uma pela floresta de liana. Vale salientar que, dadas as características deste trabalho, a intenção era considerar uma quantidade máxima de parcelas que apresentassem diferenciação de biomassa, para que esta pudesse ser detectada através de correlações entre dados radiométricos extraídos de imagens orbitais e aqueles dendrométricos descritos anteriormente. Nesse caso foi possível trabalhar com 16 parcelas, as quais foram tratadas como parte de um todo e não de estágios sucessionais específicos.

\subsection{Dados radiométricos orbitais}

Uma imagem TM/Landsat 5 de 20.07.1991 (226/ 62, nas bandas espectrais TM1-TM5 e TM7) da área de estudo foi georreferenciada através do uso de pontos de controle identificados durante trabalho de campo, cujas coordenadas geográficas foram determinadas mediante o uso de um GPS diferencial, proporcionando um erro estimado sempre menor do que o das dimensões do pixel $(30$ x $30 \mathrm{~m})$ da imagem TM. No georreferenciamento, o erro máximo admitido foi menor do que um pixel, tanto na direção horizontal quanto na vertical. O posicionamento das parcelas, bem como seus contornos, foi fornecido pela equipe responsável pelos trabalhos de campo do Anthropological Center for Training and Research on Global Environmental Change (ACT) de Indiana University dos Estados Unidos.

Uma vez concluída essa etapa, seguiu-se a transformação dos números digitais existentes nas imagens das diferentes bandas espectrais em valores de reflectância aparente, através da aplicação da equação:

$\rho_{i}=255 \frac{\pi * d^{2} *\left(L_{\min i}+\left(L_{\max i}-L_{\min i}\right)\left(N D_{i} / 255\right)\right.}{E_{i} * \cos \theta} i=1,2,3,4,5 e 7$

R. Árvore, Viçosa-MG, v.28, n.4, p.535-545, 2004 
em que:

$\rho_{\mathrm{i}}=$ a reflectância aparente na banda $\mathrm{i}$;

$\mathrm{d}=$ distância entre o Sol e a Terra (nesse caso, $\mathrm{d}=1$ );

$\mathrm{Lmin}_{\mathrm{i}}=$ radiância mínima para a banda $\mathrm{i}$, para a qual é atribuído o valor 0;

$\mathrm{Lmax}_{\mathrm{i}}=$ radiância máxima para a banda $\mathrm{i}$, para a qual é atribuído o valor 255 ;

$\mathrm{ND}_{\mathrm{i}}=$ valor do número digital na banda $\mathrm{i}$;

$\mathrm{E}_{\mathrm{i}}=$ = irradiância solar no topo da atmosfera na banda $\mathrm{i}$; e

$\theta=$ ângulo solar zenital.

As imagens de reflectância aparente resultantes foram submetidas ao programa $6 \mathrm{~S}$ de correção atmosférica (VERMOTE et al., 1997), com o objetivo de transformá-las em imagens de reflectância de superfície a partir das quais foram extraídos os dados de cada uma das 16 parcelas que foram utilizadas no estabelecimento das correlações entre esses e os dados dendrométricos (biofísicos) descritos anteriormente. Esses dados radiométricos foram determinados para cada parcela, mediante o cálculo de médias aritméticas entre os valores de reflectância de superfície extraídos de toda a extensão da parcela. Assim, um único valor de reflectância de superfície (em cada banda espectral) passou a representar uma parcela específica.

\subsection{Correlações entre dados radiométricos e biofísicos}

As correlações entre os dados radiométricos e os biofísicos foram avaliadas através de duas estratégias distintas. Na primeira, gráficos de reflectância de superfície de cada fisionomia florestal (SS1, SS2, SS3, Florestas de Terra Firme e de Liana) foram elaborados, e o posicionamento relativo entre as curvas obtidas foi estudado. Na segunda, foram determinados coeficientes de correlação entre os dados radiométricos (reflectância de superfície) e os biofísicos com o objetivo de identificar possibilidades do estabelecimento de equações de regressão linear que permitissem a elaboração de imagens classificadas que representassem a distribuição espacial de dados biofísicos específicos. Esses modelos foram elaborados a partir dos dados de 11 parcelas, uma vez que os dados referentes a 5 delas selecionadas aleatoriamente foram utilizados na validação dos modelos de regressão estabelecidos.

\section{RESULTADOS E DISCUSSÃO}

\subsection{Dados biofísicos e radiométricos}

No Quadro 1, apresentam-se os valores de cada um dos parâmetros biofísicos considerados em cada parcela, associados aos valores de reflectância de superfície extraídos das imagens orbitais.

Quadro 1 - Dados biofísicos e radiométricos de cada parcela Table 1 -Biophysical and radiometric data for each plot

\begin{tabular}{|c|c|c|c|c|c|c|c|c|c|c|c|c|}
\hline Parcela & $\begin{array}{l}\text { DAP } \\
(\mathrm{cm})\end{array}$ & $\begin{array}{c}\text { GT } \\
\left(\mathrm{m}^{2} / \mathrm{plot}\right)\end{array}$ & $\begin{array}{c}\mathrm{G} \\
\left(\mathrm{m}^{2} / \mathrm{ha}\right)\end{array}$ & $\begin{array}{c}\mathrm{H} \\
(\mathrm{m})\end{array}$ & $\begin{array}{l}\text { Rug } \\
(\mathrm{m})\end{array}$ & TM1 & TM2 & TM3 & TM4 & TM5 & TM7 & Estágio \\
\hline A004_93 & 4,304 & 0,087 & 1,74 & 5,125 & 1,992 & 2,353 & 5,490 & 4,314 & 26,667 & 12,157 & 3,137 & SS1 \\
\hline A009_93 & 0 & 0,049 & 0,97 & 3,444 & 1,848 & 2,909 & 5,784 & 5,098 & 31,046 & 17,680 & 5,490 & SS1 \\
\hline A010_92 & 0 & 0,004 & 0,42 & 3,444 & 1,755 & 2,353 & 5,098 & 4,510 & 29,150 & 15,425 & 3,791 & SS1 \\
\hline A013_93 & 12,057 & 1,03 & 6,87 & 8,574 & 4,508 & 2,366 & 4,719 & 4,392 & 27,987 & 13,294 & 3,059 & $\mathrm{SS} 2$ \\
\hline A012_93 & 18,604 & 7,38 & 49,18 & 9 & 4,355 & 2,378 & 5,025 & 4,461 & 28,211 & 13,358 & 3,398 & $\mathrm{SS} 2$ \\
\hline A006_93 & 14,375 & 2,279 & 15,19 & 11,815 & 4,554 & 2,287 & 5,131 & 4,477 & 30,163 & 15,098 & 3,398 & $\mathrm{SS} 2$ \\
\hline A007_93 & 11,889 & 1,662 & 11,08 & 9,306 & 5,124 & 2,255 & 4,706 & 4,265 & 29,755 & 14,069 & 3,382 & $\mathrm{SS} 2$ \\
\hline A005_93 & 22,749 & 3,766 & 25,11 & 12,668 & 8,71 & 2,353 & 5,458 & 4,804 & 31,340 & 15,262 & 3,562 & $\mathrm{SS} 2$ \\
\hline A010_93 & 3,732 & 0,083 & 8,29 & 4,319 & 2,979 & 2,287 & 5,490 & 4,510 & 33,137 & 16,568 & 4,216 & $\mathrm{SS} 2$ \\
\hline A011_93 & 11,526 & 2,128 & 14,18 & 8,547 & 3,375 & 2,516 & 5,327 & 4,542 & 28,791 & 14,902 & 4,020 & $\mathrm{SS} 2$ \\
\hline A014_93 & 11,011 & 1,817 & 12,11 & 7,846 & 3,759 & 2,373 & 5,275 & 4,549 & 35,020 & 15,941 & 3,765 & $\mathrm{SS} 2$ \\
\hline A001_93 & 16,225 & 2,045 & 13,58 & 11,625 & 5,103 & 2,374 & 4,860 & 4,412 & 27,696 & 12,276 & 2,577 & SS3 \\
\hline A002_93 & 13,793 & 2,055 & 13,70 & 10,768 & 5,777 & 2,340 & 4,941 & 4,360 & 25,954 & 11,987 & 2,928 & $\mathrm{SS} 3$ \\
\hline A001_92 & 21,267 & 5,882 & 26,14 & 19,257 & 7,197 & 2,231 & 4,559 & 4,069 & 27,402 & 11,299 & 1,985 & SS3 \\
\hline A008_93 & 16,216 & 4,718 & 23,59 & 12,507 & 6,973 & 2,243 & 4,657 & 3,934 & 26,471 & 11,078 & 2,206 & Firme \\
\hline A003_93 & 17,907 & 6,687 & 32,93 & 12,343 & 11 & 2,218 & 4,465 & 3,975 & 25,764 & 10,605 & 2,038 & liana \\
\hline
\end{tabular}

R. Árvore, Viçosa-MG, v.28, n.4, p.535-545, 2004 
A coluna estágio refere-se à classificação adotada por Mausel et al. (1993), fundamentando-se em seus trabalhos de campo, apresentada aqui em ordem crescente, ou seja, das classes com menor até as de maior biomassa. E interessante observar que a rugosidade do dossel é crescente à medida que se avança no estágio de desenvolvimento da cobertura vegetal. A dinâmica dos demais parâmetros segue a mesma tendência, o que corresponde a algo perfeitamente esperado, ou seja, quanto mais avançado é um estágio de desenvolvimento de um dossel florestal, são esperados valores maiores de DAP, GT, G e H. O aumento da rugosidade é mais sutil e dependente da estratificação vertical do dossel.

Considerando, então, cada um desses parâmetros biofísicos separadamente, novos escalonamentos poderiam ser propostos. No Quadro 2, apresentam-se novos escalonamentos, segundo cada um dos parâmetros biofísicos considerados.

Observa-se, nesse quadro, que o escalonamento mais próximo ao proposto por Mausel et al. (1993) foi aquele elaborado a partir dos dados da rugosidade do dossel. Optou-se, então, por estratificar os dados biofísicos em quatro classes diferentes, os quais se encontram assinalados em cores no Quadro 2. A cor vermelha foi atribuída àquele estágio inicial que continuará sendo denominado SS 1, azul para o estágio intermediário SS2, lilás para o estágio avançado SS3 e, finalmente, azul-petróleo para as florestas primá- rias, aqui simplesmente denominadas "Floresta".

Dados radiométricos (reflectância de superfície) de cada uma dessas parcelas foram extraídos das imagens e organizados segundo esse novo critério de escalonamento, originando os gráficos apresentados na Figura 2.

Comparando as curvas em cada um desses gráficos, é possível observar que na região do visível (TM1, TM2 e TM3) os estágios sucessionais apresentaram valores de reflectância de superfície muito similares entre si, enquanto na região do infravermelho é possível verificar algumas diferenças. No infravermelho próximo (TM4), o estágio SS2 apresentou valores relativamente mais elevados de reflectância de superfície do que os outros estágios, incluindo o de floresta. Seria esperado que, à medida que se verifica um aumento na rugosidade do dossel, sua reflectância nessa região espectral deveria decrescer devido ao sombreamento mútuo de um estrato ou de copas de árvores dominantes que se projetam na parte mais superior do topo do dossel (SOUSA, 1997; BERNARDES, 1996; e MORAN et al., 2000), mas essa dinâmica não foi evidente a partir da observação desses gráficos. No infravermelho médio (TM5 e TM7), as diferenças foram menores, mas os primeiros estágios sucessionais tenderam a apresentar valores de reflectância de superfície ligeiramente maiores do que os estágios mais avançados, o que é um resultado igualmente esperado, uma vez que a maior quantidade de folhas existentes

Quadro 2 - Possíveis escalonamentos segundo cada um dos parâmetros biofísicos

Table 2 - Possible successional plot rankings by each biophysical parameter

\begin{tabular}{|c|c|c|c|}
\hline Pelo DAP & Pela G & Pela H & Pela Rug \\
\hline A009_93-SS1 & A010_92-SS1 & A010_92-SS1 & A010_92-SS1 \\
\hline A010_92-SS1 & A009_93-SS1 & A009_93-SS1 & A009_93-SS1 \\
\hline A010_93-SS2 & A004_93-SS1 & A010_93-SS2 & A004_93-SS1 \\
\hline A004_93-SS1 & A013_93-SS2 & A004_93-SS1 & A010_93-SS2 \\
\hline A014_93-SS2 & A010_93-SS2 & A014_93-SS2 & A011_93-SS2 \\
\hline A011_93-SS2 & A007_93-SS2 & A011_93-SS2 & A014_93-SS2 \\
\hline A007_93-SS2 & A014_93-SS2 & A013_93-SS2 & A012_93-SS2 \\
\hline A013_93-SS2 & A001_93-SS3 & A012_93-SS2 & A013_93-SS2 \\
\hline A002_93-SS3 & A002_93-SS3 & A007_93-SS2 & A006_93-SS2 \\
\hline A006_93-SS2 & A011_93-SS2 & A002_93-SS3 & A001_93-SS3 \\
\hline A008_93 - Firme & A006_93-SS2 & A001_93-SS3 & A007_93-SS2 \\
\hline A001_93-SS3 & A008_93 - Firme & A006_93-SS2 & A002_93-SS3 \\
\hline A003_93 - Liana & A005_93-SS2 & A003_93-Liana & A008_93-Firme \\
\hline A012_93-SS2 & A001_92-SS3 & A008_93-Firme & A001_92-SS3 \\
\hline A001_92-SS3 & A003_93 - Liana & A005_93-SS2 & A005_93-SS2 \\
\hline A005_93-SS2 & $\mathrm{A} 012 \_93-\mathrm{SS} 2$ & A001_92-SS3 & A003_93-Liana \\
\hline
\end{tabular}


nos estágios mais avançados reduz a reflectância do dossel pela maior oferta de água, que nessa faixa espectral domina o comportamento espectral da vegetação (GOEL, 1988; PONZONI e DISPERATI, 1994).

Valendo-se de médias aritméticas dos dados radiométricos de cada um desses estágios sucessionais, foi elaborado outro gráfico com curvas espectrais representativas de cada estágio sucessional, as quais se encontram na Figura 3.

Devido ao baixo número de parcelas em cada estágio sucessional não foi possível aplicar qualquer procedimento estatístico para comprovar a significância das diferenças dessas curvas em cada banda espectral. Contudo, foi possível verificar o posicionamento relativo dos valores de reflectância de superfície de cada estágio. Assim, é possível constatar que todas as curvas espectrais parecem organizadas em dois grupos: um deles composto por SS1 e SS2 (Grupo 1) e outro por SS3 e Floresta (Grupo 2). Esse posicionamento é mais evidente na região do infravermelho (próximo e médio). Considerando esses dois grupos, na região do visível, mais especificamente nas bandas TM2 e TM3, o Grupo 2 apresentou os valores mais baixos de reflectância de superfície, o que concorda com o esperado no que

SS1

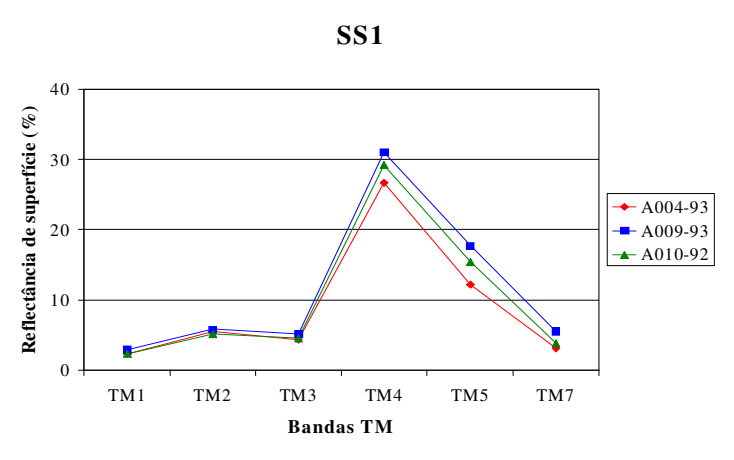

SS3

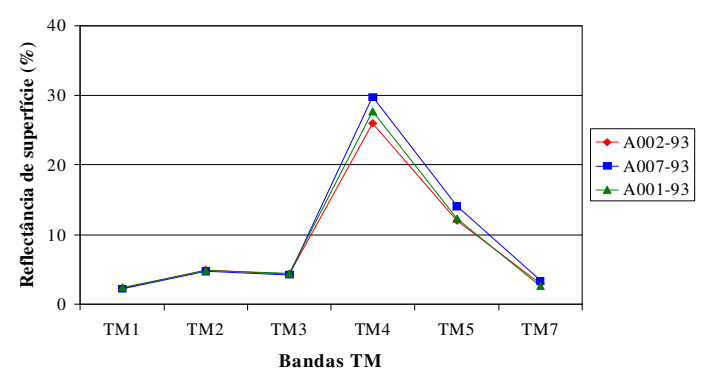

se refere ao processo de interação da radiação eletromagnética com a vegetação, uma vez que nesses estágios mais avançados é esperada uma maior quantidade de folhas e, conseqüentemente, de pigmentos fotossintetizantes, que absorvem a radiação incidente nessa faixa espectral (GOEL, 1988; KUMAR, 1974). Aliada a essa maior quantidade de pigmentos fotossintetizantes, o efeito do sombreamento não pode aqui ser desprezado, mesmo para essa faixa espectral (PONZONI, 1993). Na região do infravermelho (TM4), seria esperado um aumento na reflectância devido ao espalhamento múltiplo ocasionado pelo já mencionado aumento do número de folhas, assim como reportado por Gausman et al. (1974) e Kumar (1974) para vegetações herbácea e arbustiva, nas para formações arbóreas o sombreamento assume papel fundamental e dominante no processo de interação da radiação com o dossel, reduzindo dramaticamente sua reflectância (WALTER-SHEA, 1987; PONZONI e DISPERATI, 1994) nessa região espectral. Ponzoni et al. (2001) e Galvão et al. (2001) mencionaram o efeito da estratificação do dossel na diminuição da reflectância de superfície na região do infravermelho próximo de dosséis arbóreos de formações secundárias em uma área de estudo na Amazônia brasileira.
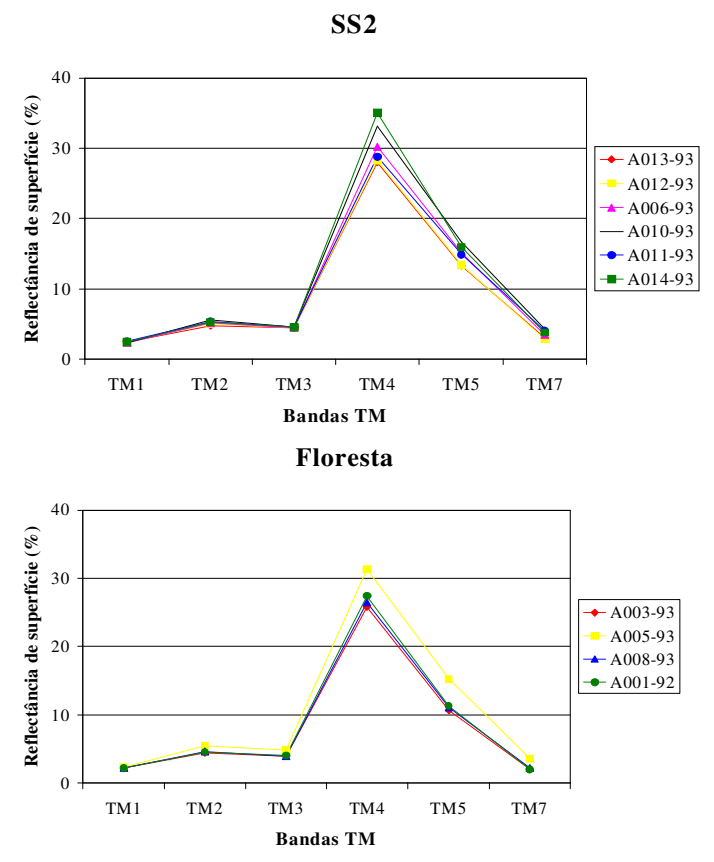

Figura 2 - Reflectância de superfície em cada estágio sucessional. Figure 2-Surface reflectance values for each successional stage.

R. Árvore, Viçosa-MG, v.28, n.4, p.535-545, 2004 


\section{Estágios sucessionais}

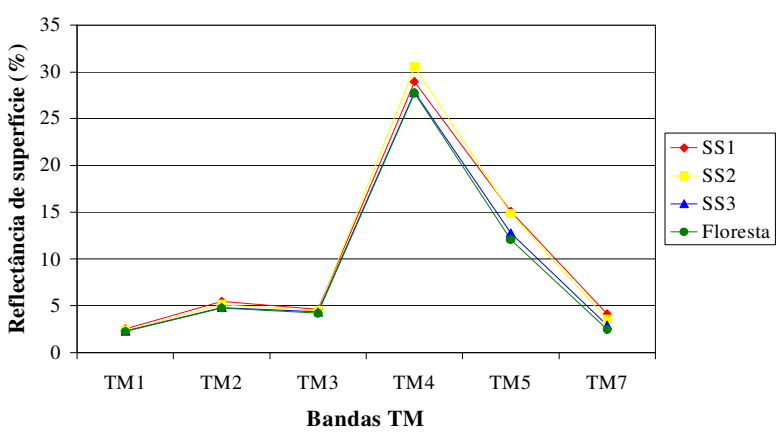

Figura 3 - Curvas espectrais dos estágios sucessionais. Figure 3 - Spectral curves of each successional classes.

Na região do infravermelho médio (TM5 e TM7), as diferenças entre as curvas foram mais evidentes, ressaltando-se que o Grupo 2 apresentou os menores valores, o que também concorda com o esperado, pelas mesmas razões já apresentadas.

Para melhor entender a relação entre os parâmetros radiométricos e os biofísicos, foram determinados coeficientes de correlação, os quais se encontram no Quadro 3.

Considerando as principais características da interação entre a vegetação e a radiação eletromagnética descritas por Goel (1988), Kumar (1974) e Ponzoni (1993), relações inversas são esperadas nas regiões do visível e do infravermelho médio e relações positivas na região do infravermelho próximo, principalmente na vegetação de porte herbáceo ou arbustivo. Contudo, nessa última região espectral há de serem considerados os aspectos já mencionados referentes ao regime de sombreamento mútuo discutido por Sousa (1997), Bernardes (1996) e Ponzoni (1993), que nas formações arbóreas acabam também invertendo essa relação.

Quadro 3 - Coeficientes de correlação dos dados radiométricos e os biofísicos

Table 3 - Correlation coefficients for both radiometric and biophysical data

\begin{tabular}{cccccc}
\hline Radiométricos & \multicolumn{5}{c}{ Biofísicos } \\
\cline { 2 - 6 } & DAP & G & GT & H & Rug \\
\hline TM1 & -.50 & -.36 & -.39 & -.52 & -.51 \\
TM2 & -.57 & -.43 & -.57 & -.66 & -.63 \\
TM3 & -.42 & -.36 & -.50 & -.55 & -.52 \\
TM4 & -.30 & -.27 & -.39 & -.38 & -.37 \\
TM5 & -.56 & -.45 & -.58 & -.64 & -.60 \\
TM7 & -.68 & -.47 & -.61 & -.76 & -.69 \\
\hline
\end{tabular}

Observando os valores desses coeficientes de correlação apresentados no Quadro 3, é possível verificar que os valores mais elevados foram encontrados nas relações com a banda TM7. Para esses dados então foram estabelecidas equações de regressão linear, tendo como variável dependente o parâmetro biofísico e, como independente, a reflectância de superfície na banda TM7. As equações resultantes foram:

$$
\begin{aligned}
& \text { DAP }=26,908-4,134 \text { TM7 } r^{2}=0,27 \\
& G=35,776-5,295 \text { TM7 } r^{2}=0,09 \\
& \text { GT }=8,754-1,789 \text { TM7 } r^{2}=0,30 \\
& H=22,047-3,849 \text { TM7 } r^{2}=0,54 \\
& \text { Rug }=12,195-2,185 \text { TM7 } r^{2}=0,40
\end{aligned}
$$

Como descrito anteriormente, essas equações foram determinadas considerando dados de 11 parcelas, uma vez que os dados de cinco delas foram utilizados para validação desses modelos de regressão estabelecidos. Na Figura 4, mostraram-se as imagens resultantes da aplicação dessas equações de regressão da parte da área de estudo, as quais serão aqui tratadas como mapas temáticos.

Comparando os mapas temáticos com a composição colorida nessa mesma Figura 4, é possível constatar visualmente uma grande correspondência entre a localização das formações secundárias e as quantidades dos parâmetros biofísicos estimadas pela aplicação dos modelos. Os modelos foram, então, validados através da utilização dos dados das cinco parcelas aleatoriamente selecionadas, cujos resultados se encontram na Figura 5.

Os gráficos apresentados na Figura 5 mostram a comparação entre os valores dados coletados em campo e aqueles estimados através dos modelos de regressão. Quanto mais próximos da linha diagonal estiverem os pontos pretos, melhor será o desempenho do modelo na estimativa do parâmetro biofísico. Verificou-se, portanto, que as estimativas da altura do dossel $(\mathrm{H})$ e da rugosidade do dossel (Rug) foram as que alcançaram melhor desempenho. Outro aspecto relevante referiu-se ao fato de que valores mais elevados de qualquer parâmetro biofísico proporcionaram estimativas mais próximas da realidade, indicando que valores de reflectância de superfície não são sensíveis à detecção de pequenos valores biofísicos.

\section{sitf}

R. Árvore, Viçosa-MG, v.28, n.4, p.535-545, 2004 


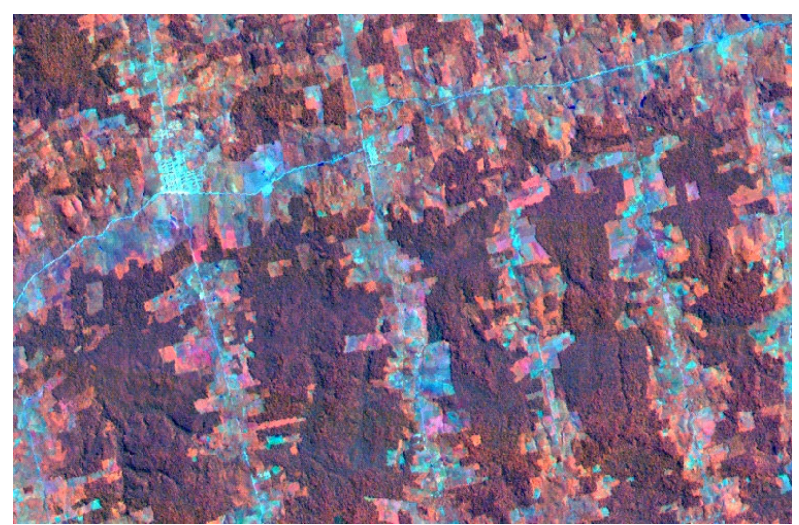

Composição colorida (TM3B, TM4R, TM5G)

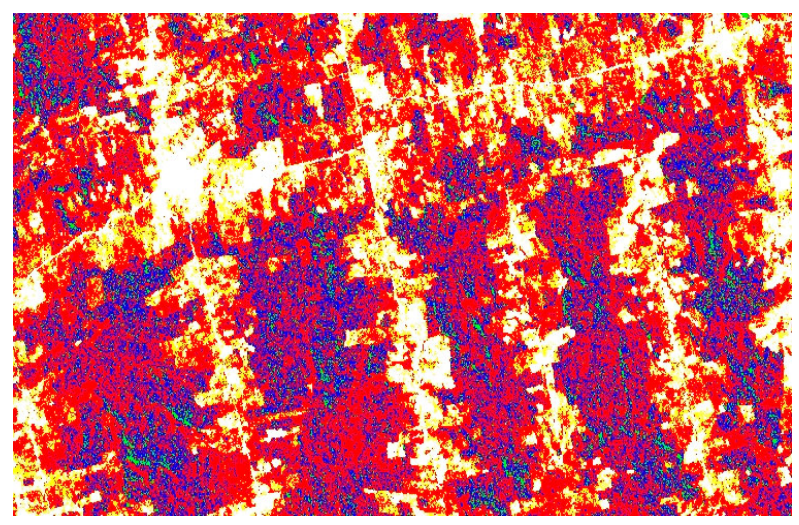

Imagem $\mathrm{G}$

0-10 $\mathrm{m}^{2} / \mathrm{ha}$ amarelo; $10-20 \mathrm{~m}^{2} /$ ha vermelho; $20-30 \mathrm{~m}^{2} / \mathrm{ha}$ azul; $30-40 \mathrm{~m}^{2} /$ ha verde; $40-50 \mathrm{~m}^{2} /$ ha verde-escuro

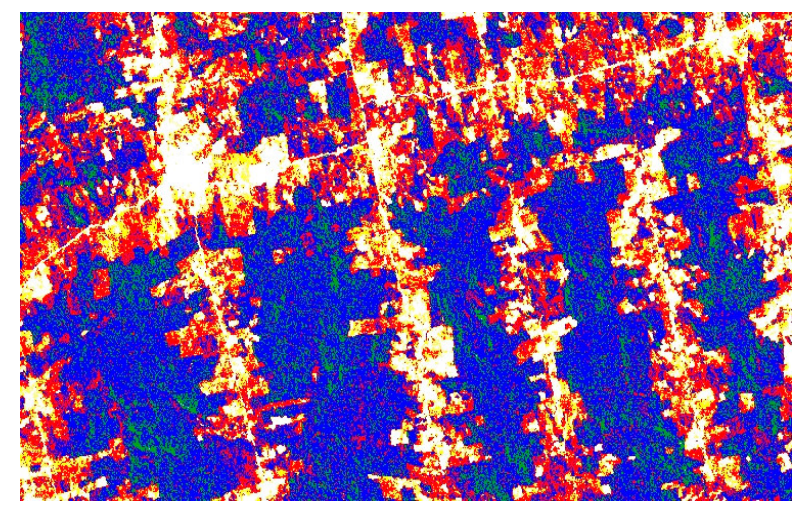

Imagem $\mathrm{H}$

0-5 m amarelo; 5-10 m vermelho; 10-15 m azul; $15-25 \mathrm{~m}$ verde

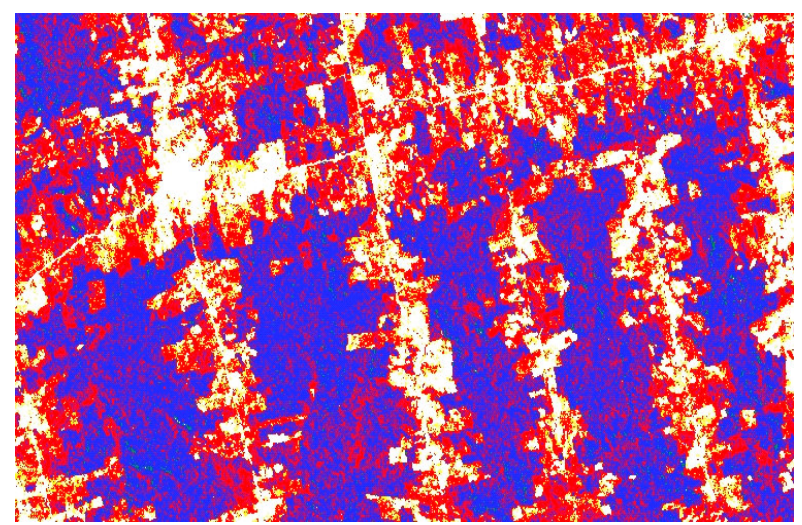

Imagem DAP

0-5 cm amarelo; $5-15 \mathrm{~cm}$ vermelho; $15-25 \mathrm{~cm}$ verde

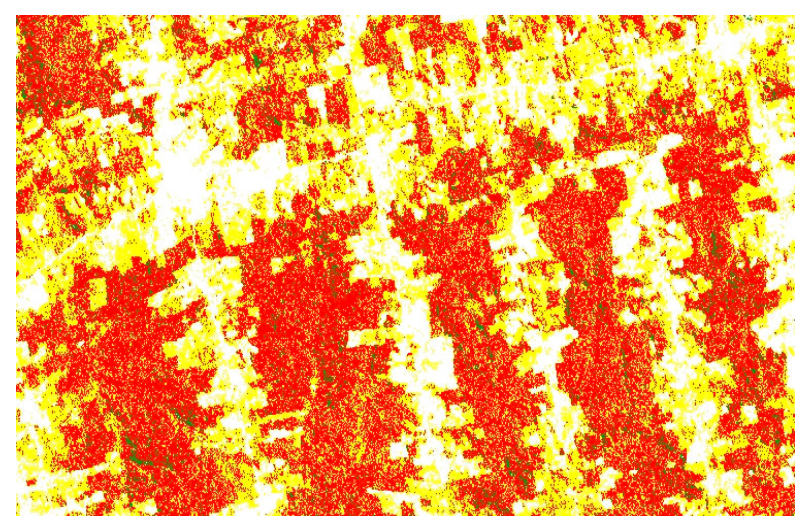

Imagem GT

0-3 $\mathrm{m}^{2}$ amarelo; 3-6 $\mathrm{m}^{2}$ vermelho; 6-9 $\mathrm{m}^{2}$ verde

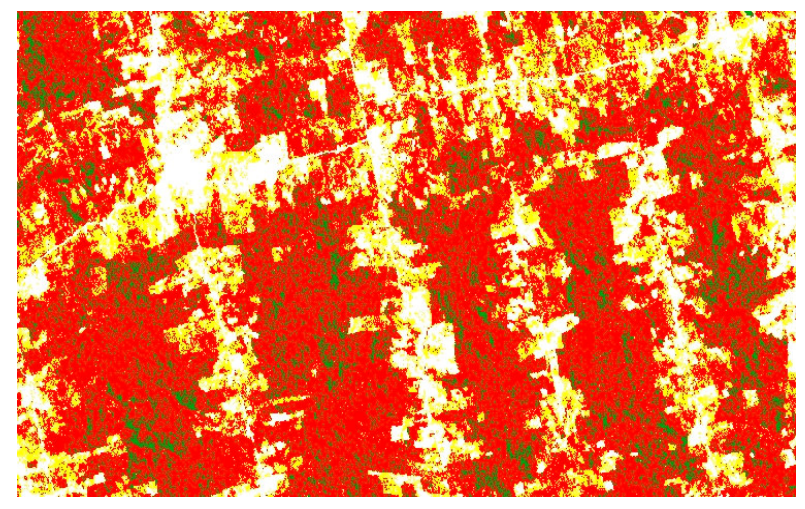

Imagem Rug

0-4 $\mathrm{m}$ amarelo; 4-8 $\mathrm{m}$ vermelho; 8 -12 $\mathrm{m}$ verde

Figura 4 - Distribuição espacial dos parâmetros biofísicos. Figure 4 - Spatial distribution of biophysical parameters.

R. Árvore, Viçosa-MG, v.28, n.4, p.535-545, 2004 


$$
\mathbf{D A P}_{\text {obs }} \mathbf{x} \mathbf{D A P}_{\text {cal }}
$$

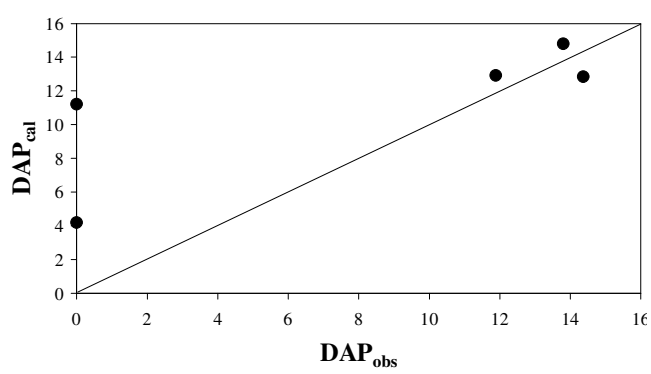

$\mathbf{G T}_{\text {obs }} \times \mathbf{G T}_{\text {cal }}$

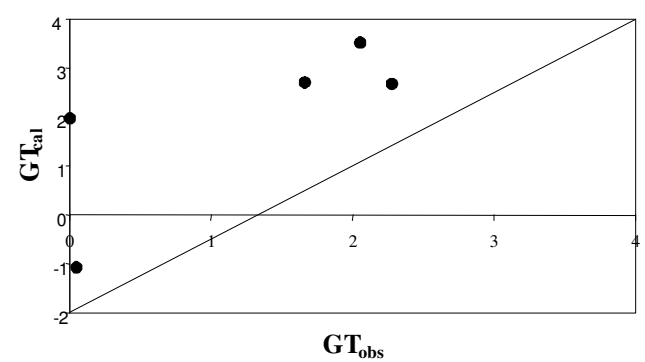

$\mathbf{G}_{\text {obs }} \times \mathbf{G}_{\text {cal }}$

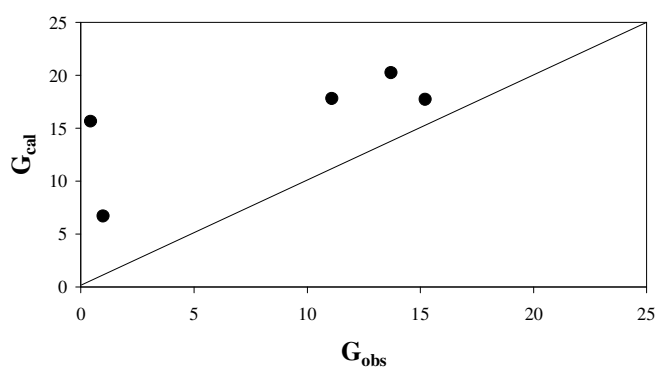

$\mathbf{H}_{\text {obs }} \times \mathbf{H}_{\text {cal }}$

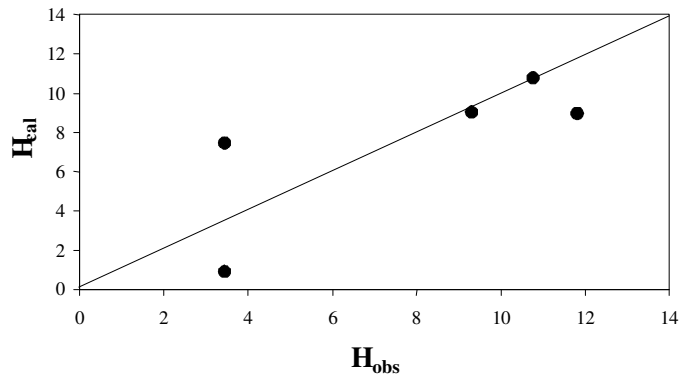

$\operatorname{Rug}_{\text {obs }} \times$ Rug $_{\text {cal }}$

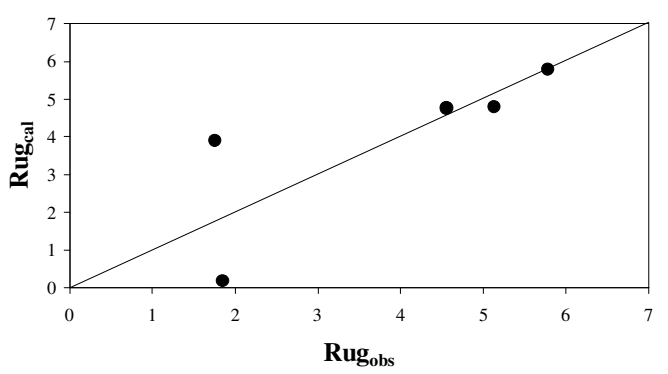

Figura 5 - Validação dos modelos de regressão.

Figure 5 - Regression models validation.

\section{CONCLUSÕES}

Na caracterização espectral de estágios sucessionais de formações arbóreas na região de Altamira, PA, observou-se que estágios mais avançados apresentaram valores mais baixos de reflectância de superfície em todas as faixas espectrais estudadas. Esse fato deve ser explicado pela maior quantidade de sombras devido à maior estratificação vertical nos estágios mais avançados.

Os coeficientes de correlação foram mais eleva- dos nas relações estabelecidas com os dados de altura $(\mathrm{H})$ e de rugosidade do dossel (Rug) com os dados radiométricos das bandas do infravermelho médio, em relação àqueles determinados a partir dos dados provenientes das bandas espectrais do visível (TM1, TM2 e TM3) e do infravermelho próximo (TM4). Tal fato redireciona os esforços na elaboração de índices que costumeiramente são estabelecidos com dados das bandas do vermelho e do infravermelho próximo.

A inspeção visual de uma composição colorida e dos mapas temáticos elaborados a partir dos mo- 
delos de regressão estabelecidos revelou correspondência positiva entre o posicionamento espacial das formações secundárias e as quantidades dos parâmetros biofísicos, indicando que a metodologia aqui explorada pode ser um excelente ponto de partida para a estimativa de dados biofísicos a partir daqueles radiométricos extraídos de imagens orbitais.

\section{AGRADECIMENTOS}

Os autores expressam seus mais sinceros agradecimentos ao Anthropological Center for Training and Research on Global Environmental Change (ACT) de Indiana University, pelo acesso aos dados; e à National Aeronautics and Space Administration (grant \#N005-334), por, através de um contrato com o ACT e o INPE, ter viabilizado financeiramente o desenvolvimento deste e de outrostrabalhos. Vale salientar que o ACT não tem responsabilidade pelos resultados e pelas conclusões aqui apresentados.

\section{REFERÊNCIAS BIBLIOGRÁFICAS}

ALVES, D. S. et al. Characterizing landscape changes in Central Rondônia using Landsat TM imagery. International Journal of Remote Sensing, v. 20, n. 14, p. 2877-2882, 1999.

BERNARDES, S. Índices de vegetação e valores de proporção na caracterização de floresta tropical primária e estádios sucessionais na área de influência da Floresta de Tapajós-Estado do Pará. 1996, 94f. Dissertação (Mestrado). Instituto Nacional de Pesquisas Espaciais (INPE), São José dos Campos, 1996.

GALVÃO, L. S.; PONZONI, F.J.; EPIPHANIO, J.C.N. Efeitos da geometria de visada sobre a determinação de índices de vegetação. In: SIMPÓSIO BRASILEIRO DE SENSORIAMENTO REMOTO, 10., 2001. Foz do Iguaçú. Anais ... Foz do Iguaçu: 2001. cdrom.

GAUSMAN, H.W. Leaf reflectance of nearinfrared. Photogrammetric Engineering, v. 40, n. 2 , p. 57-62, 1974.

GOEL, N. S. Models of vegetation canopy reflectance and their use in estimation of biophysical parameters from reflectance data. Remote Sensing Reviews, v. 4 p. 1-21, 1988.
KUMAR,R. Radiation from plantsreflection and emission: a review. West Lafayette: Purdue Research Foundation, 1994. 88p. (Research Project, 5543).

MAUSEL, P. et al. Spectral identification of successional stages following deforestation in the Amazon. Geocarto International, v. 8, n. 4, p. 61-71, 1993.

MORAN, E.F. et al. Effects of soil fertility and land-use on forest successional in Amazônia. Forest Ecology and Management, v. 139 p. 93-108, 2000.

PONZONI, F. J. Aplicação do modelo SAIL no estudo da reflectância espectral de dosséis de mudas de Eucalyptus sp. e de Aspidosperma sp.. 1993. 253 f. Tese (Doutorado). Universidade Federal do Paraná, Curitiba, 1993.

PONZONI, F. J.; DISPERATI, A. A. Aplicação do modelo SAIL no estudo da reflectância espectral de dosséis de mudas de Eucalyptus sp. e de Aspidosperma sp. Revista Floresta, v. 21, n. 1/2, p. 45-70, 1994.

PONZONI, F.J.; GALVÃO, L. S.; EPIPHANIO, J. C. N. Influência da resolução espacial sobre a quantificação de áreas não florestadas em ambiente amazônico. In: SIMPÓSIO BRASILEIRO DE SENSORIAMENTO REMOTO, 10., 2001. Foz do Iguaçú. Anais ... Foz do Iguaçu, 2001. cdrom.

PONZONI, F.J. Comportamento Espectral da Vegetação. In: MENESES, P.R.; MADEIRA NETO, J.S., (Eds). Sensoriamento remoto: reflectância de alvos naturais. Brasília: UNB/ EMBRAPA, 2001.

PONZONI F. J. et al. Coleta de dados radiométricos e biométricos e estimativas de Índice de Área Foliar em florestas implantadas de Eucalyptus spp. em Cachoeira do Manteiga (MG). São José dos Campos: Instituto Nacional de Pesquisas Espaciais, 1996. (INPE-5973-NCT/326). 
SOUSA, C.L.; PONZONI, F.J. Considerações sobre transformação de dados orbitais para estimativas do Índice de Área Foliar (IAF) In:

Simpósio Brasileiro de Sensoriamento Remoto, 8., 1996. Salvador. Anais... São José dos Campos: INPE, 1996. cdrom.

SOUSA, C.L. Uso de imagens-índice e de imagens-proporção para avaliar a quantidade de madeira em povoamentos de Pinus spp. São José dos Campos: Instituto Nacional de Pesquisas Espaciais, 1997. 111p. (INPE-6398-TDI/614).
VERMOTE, E.F. et al. Second simulation of the satellite signal in the solar spectrum, 6S: An overview., IEEE Transations Geoscience and Remote Sensing, v. 35, n 3, p. 675-686, 1997.

WALTER-SHEA, E.A. Laboratory and field measurements of leaf spectral properties and canopy architecture and their effects on canopy reflectance. 1987.154 f. (Ph.D. Thesis), University of Nebraska, Lincoln, Neb, 1987. 